\title{
Maximum Lesion-To-Contralateral Normal Brain Tissue Ratio of 11C-Methionine PET as a Prognostic Imaging Biomarker for Newly Diagnosed and Untreated Astrocytic Glioma
}

Kosuke Nakajo ( $\square$ kousuke19841984@yahoo.co.jp )

Osaka City University

Takehiro Uda

Osaka City University

Toshiyuki Kawashima

Osaka City University

Yuzo Terakawa

Hokkaido Ohno Memorial Hospital

Kenichi Ishibashi

Osaka City General Hospital

Naohiro Tsuyuguchi

kinki university

Yuta Tanoue

Osaka City University

Atsufumi Nagahama

Osaka City University

Saya Koh

Osaka City University

Hiroshi Uda

Osaka City University

Tsuyoshi Sasaki

Osaka City University

Kenji Ohata

Osaka City University

Yonehiro Kanemura

Osaka National Hospital

Takeo Goto

Osaka City University 


\section{Research Article}

Keywords: 11C-methionine, $\mathrm{PET}$, astrocytic glioma, IDH, L/N ratio

Posted Date: July 27th, 2021

DOI: https://doi.org/10.21203/rs.3.rs-729587/v1

License: (c) (i) This work is licensed under a Creative Commons Attribution 4.0 International License. Read Full License 


\section{Abstract}

Purpose: This study aimed whether the uptake of amino tracer positron emission tomography (PET) can be used as an additional imaging biomarker to estimate the prognosis of glioma.

Methods: Participants comprised 56 adult patients with newly diagnosed and untreated World Health

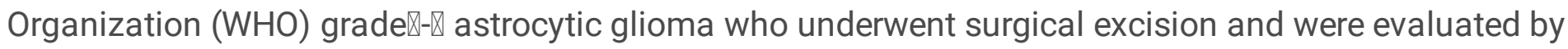
11C-methionine PET prior to the surgical excision at Osaka City University Hospital from July 2011 to March 2018. Clinical and imaging studies were retrospectively reviewed based on medical records at our institution.

Results: Preoperative Karnofsky Performance Status (KPS) only influenced progression-free survival (PFS) (hazard ratio [HR] 0.20; 95\% confidence interval [CI] 0.10-0.41, $p<0.0001$ ), whereas histology (anaplastic astrocytoma: HR 5.30, 95\% Cl 1.23-22.8, $p=0.025$; glioblastoma: HR 11.52, 95\% Cl 2.27-58.47, $p=0.0032$ ), preoperative KPS $\geq 80$ (HR $0.23,95 \% \mathrm{Cl} 0.09-0.62, p=0.004)$, maximum lesion-to-contralateral normal brain tissue (LN max) $\geq 4.03$ (HR $0.24,95 \% \mathrm{Cl} 0.08-0.71, p=0.01)$, and isocitrate dehydrogenase (IDH) status (HR $14.06,95 \% \mathrm{Cl} 1.81-109.2, p=0.011)$ were factors influencing overall survival $(O S)$ in multivariate Cox regression. OS was shorter in patients with $L N \max \geq 4.03$ (29.3 months) than in patients with LN max $<4.03$ (not reached; $p=0.03$ ). OS differed significantly between patients with $I D H$ mutant/LN max $<4.03$ and patients with IDH mutant/LN $\max \geq 4.03$.

Conclusions: LN max using 11C-methionine PET may be used in prognostic markers for newly identified

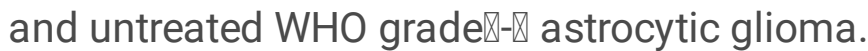

\section{Introduction}

Gliomas are the second most common primary brain tumors according to the 2012-2016 Central Brain Tumor Registry of the United States ${ }^{1}$. Approximately $48.3 \%$ of primary malignant brain tumors are glioblastomas, $16.7 \%$ are other astrocytomas, and $4.5 \%$ are oligodendrogliomas ${ }^{1}$. Overall incidence rates for diffuse astrocytoma, anaplastic astrocytoma, and oligodendroglioma have been decreasing recently, but overall incidence rates for glioblastoma have remained stable ${ }^{2}$.

Although magnetic resonance imaging (MRI) has been one of the basic and less-invasive imaging modalities used in the management of glioma, brain PET imaging has recently been recommended ${ }^{3,4}$. We have previously reported a positive correlation between WHO grade and accumulation of $11 \mathrm{C}$-methionine among astrocytomas, but that study did no analyze the relationship with prognosis ${ }^{5}$. Additional analysis was thus performed in the current study. Moreover, the clinical studies investigating the relationship between molecular analysis and uptake of amino acid PET in glioma patients are sparse, and detailed prognostic analyses of associations with molecular profiles and 11C-methionine PET uptake in glioma 
patients have not been fully completed. This study aimed to evaluate the association between $11 \mathrm{C}$ methionine uptakes, gene status, and prognosis in cases of newly diagnosed and untreated adult astrocytic glioma.

\section{Methods}

\section{Patients}

From July 2011 to March 2018, there were 66 adult patients and two patients under 18 years old with newly diagnosed and untreated WHO grade $\mathrm{W}-\mathrm{Q}$ glioma who underwent surgical tumor resection and preoperative $11 \mathrm{C}$-methionine PET examination, as previously reported ${ }^{5}$. From this previous cohort, we included adult astrocytic glioma patients with IDH mutated- TERT promoter wild-type, or those with IDH wild-type in the present study. Finally, a total of 56 patients with astrocytic tumor were included in the present cohort. The 56 patients were comprised of 36 male and 20 female patients, with a mean age of 54.0 years (range, 21-82 years). All 11C-methionine PET was performed within one month prior to tumor resection in glioblastoma patients and within six months in patients with lower-grade glioma.

Pathological diagnosis was determined according to the 2016 WHO classification for central nervous system tumors ${ }^{6}$. This study was approved by the institutional review boards at the Graduate School of Medicine, Osaka City University (approval numbers: 2047 and 2020 - 115), and Osaka National Hospital (approval number: 0713). Written informed consent was obtained from all individual participants included in this study. This study was complied with all tenets of the Declaration of Helsinki.

\section{C-methionine PET}

An Eminence B PET scanner (Shimadzu, Kyoto, Japan) or Biograph-16 PET scanner (Siemens, Bon, Germany) was used for $11 \mathrm{C}$-methionine PET, according to previously reported procedures ${ }^{5,7}$. Mean and maximum lesion-to-contralateral normal brain tissue $(\mathrm{L} / \mathrm{N})$ ratios were determined by dividing the tumor standardized uptake value by the mean standardized uptake value of the normal contralateral region of the brain, as previously reported ${ }^{5}$.

\section{Genetic Analysis}

Genetic analysis was performed as previously described ${ }^{5}$. Genomic DNA was extracted from surgically resected tumor specimens using the DNeasy Blood \& Tissue Kit (Qiagen, Valencia, CA, USA) or NucleoSpin Tissue (Machery-Nagel, Duren, Germany). Hotspot mutations of IDH1/2 (codon 132 of IDHI and codon 172 of IDH2) and TERT promoter (termed C228 and C250) were examined using Sanger sequencing with a 3130xLGenetic Analyzer (Thermo Fisher Scientific, Waltham, MA, USA) and Big-Dye ${ }^{\circledR}$ Terminator v1.1 Cycle Sequencing Kit (Thermo Fisher Scientific, Waltham, MA, USA).

\section{Survival Times}

Progression-free survival (PFS) was defined as the time in months between evaluation with $11 \mathrm{C}$ methionine PET and tumor progression according to the Response Assessment in Neuro-oncology 
working group ${ }^{8}$. Overall survival was defined as the time in months between evaluation with $11 \mathrm{C}$ methionine PET and death.

\section{Statistical Analysis}

Patients were subdivided into several groups on the basis of age ( $\geq 70$ or $<70$ years), preoperative KPS( $\geq 80$ or $<80)$, LN mean( $\geq 2.46$ or $<2.46)$, LN $\max (\geq 4.03$ or $<4.03$ ), and extent of resection (biopsy or partial removal, $<90 \%$; subtotal/gross total removal, $\geq 90 \%$ ) for statistical analysis.

To compare the patients background characteristics of each group classified according to IDH status or LN max or both, we performed statistical analysis using Pearson's chi-square test. PFS and OS were analyzed using the Kaplan-Meier method. Survival date were evaluated using uni- and multivariate Cox regression analyses. The stepwise method was used to evaluate PFS and OS multivariate Cox regression analyses. Statistical significance was defined at the level of $p<0.05$. All statistical analyses were conducted using EZR software (Saitama Medical Center, Jichi Medical University, Saitama, Japan) ${ }^{9}$.

\section{Results}

\section{Patient Characteristics}

Patient characteristics are summarized in Table 1. Majority of patients with IDH mutant astrocytoma showed lower-grade astrocytoma especially diffuse astrocytoma, whereas the majority of patients with IDH wild-type astrocytoma had high-grade glioma, especially glioblastoma $(p=0.009)$. Median LN mean and LN max were 2.46, and 4.03, respectively. Approximately $70 \%$ of patients with LN max $\geq 4.03$ showed glioblastoma, whereas nearly $60 \%$ of patients with LN max $<4.03$ showed diffuse astrocytoma $(p<$ 0.0001). According to IDH status/LN max classification, 10 patients with diffuse astrocytoma, one patient with anaplastic astrocytoma, and one patient with glioblastoma were classified into the IDH mutant with LN max $<4.03$ group. One patient with anaplastic astrocytoma, and two patients with glioblastoma were classified into the IDH mutant with LN max $\geq 4.03$ group. Seven patients with diffuse astrocytoma, five patients with anaplastic astrocytoma, and four patients with glioblastoma were classified into the IDH wild-type with LN max $<4.03$ group. Two patients with diffuse astrocytoma, five patients with anaplastic astrocytoma, and 18 patients with glioblastoma were classified into the IDH wild-type with LN max $\geq 4.03$ group.

\section{Uni- and Multivariate Analyses for PFS and OS}

In univariate analysis, age, enhancement on MRI, preoperative KPS, histology, IDH status, and TERT promoter status influenced PFS, whereas age, enhancement on MRI, preoperative KPS, LN mean, LN max, histology, and IDH status influenced OS (Table 2, Fig. 1). In multivariate Cox regression analysis, preoperative KPS only influenced PFS (HR 0.20,95\%Cl 0.1-0.41, $p<0.0001$ ), whereas histology (anaplastic astrocytoma: HR 5.3, 95\%Cl 1.23-22.8, $p=0.025$; glioblastoma: HR 11.52, 95\%Cl 2.27-58.47, $p=.0032)$, preoperative KPS $\geq 80(\mathrm{HR} 0.23,95 \% \mathrm{Cl} 0.09-0.62, p=0.004), \mathrm{LN} \max \geq 4.03(\mathrm{HR} 0.24,95 \% \mathrm{Cl}$ 
$0.08-0.71, p=0.01)$, and IDH status (HR 14.06, 95\% Cl 1.81-109.2, $p=0.011)$ were influential factors on OS (Table 3).

Median PFS in patients with diffuse astrocytoma, anaplastic astrocytoma, and glioblastoma were 37.2 months, 9.6 months, and 4.7 months, respectively $(p=0.0003$, Table 2$)$. Median OS was more favorable in patients with preoperative KPS $\geq 80$ (83.3 months) than in patients with preoperative KPS $<80$ (12.6 months, $p<0.0001$; Table 2, Fig. 2a). Median OS was more favorable in patients with diffuse astrocytoma, anaplastic astrocytoma, and glioblastoma were not applicable, 27.1 months, and 20.5 months, respectively $(p<0.0001$, Table 2 , Fig. 2 b). Median OS was more favorable in patients with IDH mutation than that in patients with IDH wild-type (not reached vs. 26.1 months, respectively, $p<0.0001$, Fig. 2c). Furthermore, OS appeared shorter in patients with $L N \max \geq 4.03$ (29.3 months) than in patients with LN max $<4.03$ (not reached, $p=0.03$; Fig. 2d).

\section{OS in Patients Classified According to the IDH Status/LN $\max$ (Fig. 3)}

Median OSs in patients with IDH mutant/LN max $<4.03$, IDH mutant/LN max $\geq 4.03$, IDH wild-type/LN max $<4.03$, and IDH wild-type/LN max $\geq 4.03$ were not applicable ( $95 \% \mathrm{Cl}$, NA-NA), 30.1 (95\%Cl, 30.1-NA), $20.5(95 \% \mathrm{Cl}, 7.4-52.3)$ and $27.1(95 \% \mathrm{Cl}, 12.6-39.8)$ months, respectively $(p=0.001)$. A significant difference in OS was seen between patients with IDH mutant/LN max $<4.03$ and those with IDH mutant/LN max $\geq 4.03(p=0.034)$, although no significant difference in OS was seen between patients with IDH mutant/LN max $\geq 4.03$ and those with IDH wild-type/LN $\max <4.03(p=0.40)$, or between patients with $I D H$ wild-type/LN max $<4.03$ and those with IDH wild-type/LN $\max \geq 4.03(p=0.84)$.

\section{Discussion}

The revised WHO 2016 classification of the central nervous system tumor requires the pathological diagnosis with molecular analysis to reach a diagnosis of glioma ${ }^{6}$. This molecular information has been said to correlate with prognosis, whereas there is still a matter of debate whether imaging biomarkers help estimation of prognosis. Although MRI remains the gold standard for diagnosing glioma, its role in estimating prognosis is limited ${ }^{10}$. On the other hand, $11 \mathrm{C}$-methionine PET using amino tracer might be useful to detect the tumor, predict the grade or genetic status or both ${ }^{5,11-14}$, and distinguish tumor recurrence from radiation necrosis ${ }^{15-17}$ in glioma patients. However, relatively few reports have investigated the relationship between the uptake of amino tracer using PET and prognosis in glioma. Moreover, reports investigating prognosis of glioma patients in association with molecular analysis and PET in glioma have been limited ${ }^{18-22}$. Thus, our goal in the present study was to determine whether 11Cmethionine PET can be used as an additional imaging biomarker of prognosis.

In the present study, we excluded patients with oligodendroglioma, or those with IDH mutated- TERT promoter mutated, or both because oligodendroglioma is considered to show better prognosis than astrocytoma and is often accompanied by both IDH and TERT promoter mutations. Although TERT 
promoter mutation is often seen in oligodendroglioma and primary glioblastoma, prognoses differ markedly between oligodendroglioma and glioblastoma ${ }^{23,24}$. An argument has also been made regarding the association between uptake of $11 \mathrm{C}$-methionine and oligodendroglioma ${ }^{14,25-28}$. We have previously reported a positive correlation between WHO grade and the accumulation of $11 \mathrm{C}$-methionine among astrocytomas, and a statistically higher uptake of 11C-methionine in oligodendroglioma than in diffuse astrocytoma ${ }^{5}$. Median survival rates in patients with diffuse astrocytoma, anaplastic astrocytoma, and glioblastoma in this study were 37.2 months, 9.6 months, and 4.7 months for PFS, and not reached, 27.1 months, and 20.5 months for OS, respectively. Reuss et al. reported that 139 of 152 patients with diffuse astrocytoma diagnosed according to the WHO 2007 classification of the central nervous system tumors showed $I D H$ mutant diffuse astrocytoma, whereas more than half of patients with diffuse astrocytoma were $I D H$ wild-type in our cohort ${ }^{29}$. Minniti et al. reported that $I D H$ mutant anaplastic astrocytoma was found in $56 \%$ of their anaplastic astrocytoma patients. OS in patients with $I D H$ wild-type was 2.8 years ${ }^{30}$. The relatively shorter PFS and OS of patients with diffuse astrocytoma and anaplastic astrocytoma in the current study were probably attributable to the fact that the present cohort included more patients with IDH wild-type astrocytoma than the previous study. On the other hand, Wakabayashi et al. reported that the median OS in patients with glioblastoma who received Stupp's regimen was 20.3 months ${ }^{31}$, similar to our result in the current study.

Brain PET imaging has recently been recommended for use in addition to MRI in the management of glioma ${ }^{3,4}$. Takano et al. reported that PFS was worse with $L N \max \geq 2.0$ than with $L N \max <2.0$ using $11 \mathrm{C}$-methionine PET among patients with untreated, lower-grade, non-enhancing gliomas ${ }^{32}$.

Discrimination of high-grade glioma from low-grade glioma is usually difficult using MRI alone prior to tumor resection in patients with non-enhancing, lower-grade glioma, so we considered wthether 11Cmethionine PET can be used to predict the prognosis of glioma. However, we could not find significant differences in PFS between astrocytoma patients with $L N \max \geq 4.03$ and $L N$ max $<4.03$ or between those with LN mean $\geq 2.46$ and LN mean $<2.46$ in the current study.

Recently, some reports have investigated the relationship between prognosis from molecular analysis and uptake of PET using 18F-fluoro-ethyl-tyrosine (18F-FET) PET $^{18-20,33}$ and 3,4-dihydroxy-6-18F-fluoro-ethylL-phenylalanine (18F-FDOPA) PET ${ }^{34}$. Galldiks et al. in a study of photopenic IDH mutant gliomas reported that glioma with 18F-FET accumulation below the level of background healthy brain showed unfavorable outcomes, and thus should be treated more actively ${ }^{19}$. The utility of dynamic 18 F-FET PET has also been reported ${ }^{20}$. Suchorska et al. reported that longer minimal time-to-peak analysis using 18F-FET PET was associated with a favorable prognosis in $I D H$ mutant astrocytomas ${ }^{20}$. A time-to-peak analysis $\geq 25$ min was associated with longer PFS and OS in patients with $I D H$ wild-type high-grade astrocytoma according to Bauer et $\mathrm{al}^{33}$. Kunz et al. reported homogeneous decreases in intratumoral uptake of 18F-FET over time as a factor associated with poor prognosis in non-enhancing glioma ${ }^{18}$. Using continuous measures of 18F-FDOPA PET, Patel et al. reported LN max and age as prognostic factors for OS in WHO grade $\mathbb{X}-\mathbb{X}$ gliomas, and that IDH or MGMT status did not correlate with uptake of 18F-FDOPA. In this study, we 
concluded that patients with LN max $\geq 4.03$ displayed unfavorable OS compared to patients with LN max $<4.03$ among patients with WHO grade囚- $\mathrm{Z}$ astrocytoma. We also concluded that patients with $\mathrm{LN}$ $\max \geq 4.03$ showed unfavorable OS compared those with $L N$ max $<4.03$ among patients with WHO grade $\mathbb{-} \mathrm{X} I D H$ mutant astrocytoma, although no significant difference in OS was evident between $I D H$ wild-

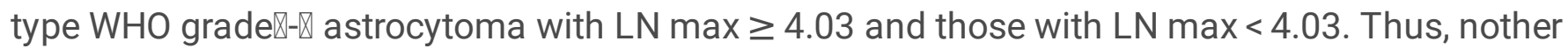
molecular imaging markers might be needed to estimate prognosis in $I D H$ wild-type astrocytoma.

Some limitations need to be considered for the current study. First, the relatively small cohort of the current study might have influenced statistical analyses. For example, TERT promoter status did not influence OS in our cohort, although Arita et al. reported tthe usefulness of TERT promoter status in addition to the $I D H$ status $^{35}$. Further study with a larger cohort is thus needed to assess the correlation between prognosis and molecular/imaging biomarkers with amino-tracer PET in patients with astrocytoma. Second, we did not take volumetric analyses into consideration in the current study, although some reports have suggested that metabolic tumor volume did not correlate with survival outcomes $^{18,20,33,34,36}$.

\section{Conclusion}

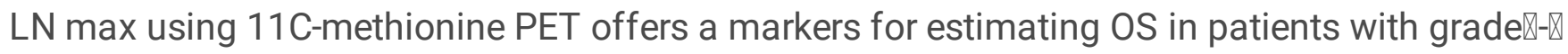
astrocytoma. LN max can also be used as a prognostic imaging biomarker to estimate OS in addition to $\mathrm{IDH}$ status in $\mathrm{IDH}$-mutated astrocytoma.

\section{Abbreviations And Acronyms}

PET: positron emission tomography; WHO: World Health Organization; KPS: Karnofsky Performance Status; PFS: progression-free survival; HR: hazard ratio; Cl: confidence interval; TERT: telomerase reverse transcriptase; LN: lesion-to-contralateral normal brain tissue; IDH: isocitrate dehydrogenase; OS: overall survival; MRI: magnetic resonance imaging ; 18F-FET: 18F-fluoro-ethyl-tyrosine; 18F-FDOPA: 3,4dihydroxy-6-18F-fluoro-ethyl-L-phenylalanine

\section{Declarations}

\section{Acknowledgements}

We would like to thank Drs. Susumu Shiomi and Shigeaki Higashiyama for their cooperation with this study, and the technologists, especially Takashi Yamanaga and Hideki Kawabata, at the Central Radiology Department, School of Medical Sciences of Osaka City University, for their support with PET. We also wish to thank Tomoko Shofuda, Ema Yoshioka, and Daisuke Kanematsu, at the National Hospital Organization Osaka National Hospital for genetic analysis.

\section{Author contributions}


K.N.: Conceptualization, Investigation, Writing-original draft. T.U.: Investigation, Supervision. T.K.: Writingoriginal draft. Y.T.: Investigation, Supervision. K.I.: Investigation, Supervision. N.T.: Conceptualization, Investigation, Supervision. Y.T.: Writing-original draft. A.N.: Writing-original draft. H.U.: Writing-original draft. S.K.: Writing-original draft. T.S.: Writing-original draft. K.O.: Supervision. Y.K.: Investigation, Resources, Supervision. T.G.: Supervision.

\section{Funding}

No funding was received for this research.

\section{Availability of date and materials.}

The date in the current study are available from the corresponding author on reasonable request.

\section{Conflicts of interest}

No funds were received in support of this work. No benefits in any form have been or will be received from any commercial party related directly or indirectly to the subject of this manuscript.

\section{Consent to participate}

Patient informed consents were waived due to the retrospective nature of the study.

\section{Consent for publication}

All authors have approved the manuscript and agree with publication.

\section{Ethical approval}

This study was approved by the institutional review boards at the Graduate School of Medicine, Osaka City University (approval numbers: 2047 and 2020-115), and Osaka National Hospital (approval number: 0713).

\section{References}

1. Ostrom, Q. T. et al. CBTRUS Statistical Report: Primary Brain and Other Central Nervous System Tumors Diagnosed in the United States in 2012-2016. Neuro-oncology 21, v1-v100, doi:10.1093/neuonc/noz150 (2019).

2. Forjaz, G. et al. An updated histology recode for the analysis of primary malignant and nonmalignant brain and other central nervous system tumors in the Surveillance, Epidemiology, and End Results Program. Neuro-oncology advances, 3, vdaa175 https://doi.org/10.1093/noajnl/vdaa175 (2021).

3. Albert, N. L. et al. Response Assessment in Neuro-Oncology working group and European Association for Neuro-Oncology recommendations for the clinical use of PET imaging in gliomas. Neurooncology, 18, 1199-1208 https://doi.org/10.1093/neuonc/now058 (2016). 
4. Law, I. et al. Joint EANM/EANO/RANO practice guidelines/SNMMI procedure standards for imaging of gliomas using PET with radiolabelled amino acids and [(18)F]FDG: version 1.0. European journal of nuclear medicine and molecular imaging, 46, 540-557 https://doi.org/10.1007/s00259-018-42079 (2019).

5. Nakajo, K. et al. Diagnostic performance of 11C-methionine PET in newly diagnosed and untreated glioma based on the revised WHO 2016 classification. World neurosurgery, doi:10.1016/j.wneu.2021.01.012 (2021).

6. Louis, D. N. et al. The 2016 World Health Organization Classification of Tumors of the Central Nervous System: a summary. Acta neuropathologica, 131, 803-820 https://doi.org/10.1007/s00401016-1545-1 (2016).

7. Tsuyuguchi, N., Terakawa, Y., Uda, T., Nakajo, K. \& Kanemura, Y. Diagnosis of Brain Tumors Using Amino Acid Transport PET Imaging with (18)F-fluciclovine: A Comparative Study with L-methyl(11)C-methionine PET Imaging. Asia Oceania journal of nuclear medicine \& biology, 5, 85-94 https://doi.org/10.22038/aojnmb.2017.8843 (2017).

8. van den Bent, M. J. et al. Response assessment in neuro-oncology (a report of the RANO group): assessment of outcome in trials of diffuse low-grade gliomas. Lancet Oncol, 12, 583-593 (2011).

9. Kanda, Y. Investigation of the freely available easy-to-use software 'EZR' for medical statistics. Bone marrow transplantation, 48, 452-458 https://doi.org/10.1038/bmt.2012.244 (2013).

10. Kanazawa, T. et al. Imaging scoring systems for preoperative molecular diagnoses of lower-grade gliomas. Neurosurgical review, 42, 433-441 https://doi.org/10.1007/s10143-018-0981-x (2019).

11. Okita, Y. et al. The association between (11)C-methionine uptake, IDH gene mutation, and MGMT promoter methylation in patients with grade II and III gliomas. Clinical radiology, 75, 622-628 https://doi.org/10.1016/j.crad.2020.03.033 (2020).

12. Falk Delgado, A. \& Falk Delgado, A. Discrimination between primary low-grade and high-grade glioma with (11)C-methionine PET: a bivariate diagnostic test accuracy meta-analysis. The British journal of radiology, 91, 20170426 https://doi.org/10.1259/bjr.20170426 (2018).

13. Nariai, T. et al. Usefulness of L-[methyl-11C] methionine-positron emission tomography as a biological monitoring tool in the treatment of glioma. Journal of neurosurgery, 103, 498-507 https://doi.org/10.3171/jns.2005.103.3.0498 (2005).

14. Shinozaki, N. et al. Discrimination between low-grade oligodendrogliomas and diffuse astrocytoma with the aid of $11 \mathrm{C}$-methionine positron emission tomography. Journal of neurosurgery, 114, 16401647 https://doi.org/10.3171/2010.11.Jns10553 (2011).

15. Terakawa, Y. et al. Diagnostic accuracy of $11 \mathrm{C}$-methionine PET for differentiation of recurrent brain tumors from radiation necrosis after radiotherapy. Journal of nuclear medicine: official publication, Society of Nuclear Medicine, 49, 694-699 https://doi.org/10.2967/jnumed.107.048082 (2008).

16. Tsuyuguchi, N. et al. Methionine positron emission tomography of recurrent metastatic brain tumor and radiation necrosis after stereotactic radiosurgery: is a differential diagnosis possible? Journal of neurosurgery, 98, 1056-1064 https://doi.org/10.3171/jns.2003.98.5.1056 (2003). 
17. Van Laere, K. et al. Direct comparison of 18F-FDG and 11C-methionine PET in suspected recurrence of glioma: sensitivity, inter-observer variability and prognostic value. European journal of nuclear medicine and molecular imaging, 32, 39-51 https://doi.org/10.1007/s00259-004-1564-3 (2005).

18. Kunz, M. et al. Dynamic 18F-FET PET is a powerful imaging biomarker in gadolinium-negative gliomas. Neuro-oncology, 21, 274-284 https://doi.org/10.1093/neuonc/noy098 (2019).

19. Galldiks, N. et al. Photopenic defects on O-(2-[18F]-fluoroethyl)-L-tyrosine PET: clinical relevance in glioma patients. Neuro-oncology, 21, 1331-1338 https://doi.org/10.1093/neuonc/noz083 (2019).

20. Suchorska, B. et al. Identification of time-to-peak on dynamic $18 \mathrm{~F}-\mathrm{FET}$-PET as a prognostic marker specifically in IDH1/2 mutant diffuse astrocytoma. Neuro-oncology, 20, 279-288 https://doi.org/10.1093/neuonc/nox153 (2018).

21. Bauer, E. K. B. et al. 2020 Prediction of survival in patients with IDH-wildtype astrocytic gliomas using dynamic 0-(2-[(18)F]-fluoroethyl)-L-tyrosine PET. European journal of nuclear medicine and molecular imaging 47, 1486-1495, doi:10.1007/s00259-020-04695-0 (2020).

22. Tatekawa, H. et al. Maximum Uptake and Hypermetabolic Volume of 18F-FDOPA PET Estimate Molecular Status and Overall Survival in Low-Grade Gliomas: A PET and MRI Study. Clinical nuclear medicine, 45, e505-e511 https://doi.org/10.1097/rlu.0000000000003318 (2020).

23. Arita, H. et al. Upregulating mutations in the TERT promoter commonly occur in adult malignant gliomas and are strongly associated with total 1p19q loss. Acta neuropathologica, 126, 267-276 https://doi.org/10.1007/s00401-013-1141-6 (2013).

24. Killela, P. J. et al. TERT promoter mutations occur frequently in gliomas and a subset of tumors derived from cells with low rates of self-renewal. Proceedings of the National Academy of Sciences of the United States of America, 110, 6021-6026 https://doi.org/10.1073/pnas.1303607110 (2013).

25. Iwadate, Y., Shinozaki, N., Matsutani, T., Uchino, Y. \& Saeki, N. Molecular imaging of 1p/19q deletion in oligodendroglial tumours with $11 \mathrm{C}$-methionine positron emission tomography. Journal of neurology, neurosurgery, and psychiatry, 87, 1016-1021 https://doi.org/10.1136/jnnp-2015-311516 (2016).

26. Kebir, S. et al. Comparison of L-Methyl-11C-Methionine PET With Magnetic Resonance Spectroscopy in Detecting Newly Diagnosed Glioma. Clinical nuclear medicine, 44, e375-e381 https://doi.org/10.1097/rlu.0000000000002577 (2019).

27. Saito, T. et al. 11C-methionine uptake correlates with combined $1 \mathrm{p}$ and $19 \mathrm{q}$ loss of heterozygosity in oligodendroglial tumors. AJNR. American journal of neuroradiology, 34, 85-91 https://doi.org/10.3174/ajnr.A3173 (2013).

28. Takei, H. et al. Usefulness of positron emission tomography for differentiating gliomas according to the 2016 World Health Organization classification of tumors of the central nervous system. Journal of neurosurgery, 1-10 https://doi.org/10.3171/2019.5.Jns19780 (2019).

29. Reuss, D. E. et al. ATRX and IDH1-R132H immunohistochemistry with subsequent copy number analysis and IDH sequencing as a basis for an "integrated" diagnostic approach for adult 
astrocytoma, oligodendroglioma and glioblastoma. Acta neuropathologica, 129, 133-146 https://doi.org/10.1007/s00401-014-1370-3 (2015).

30. Minniti, G. et al. IDH1 mutation and MGMT methylation status predict survival in patients with anaplastic astrocytoma treated with temozolomide-based chemoradiotherapy. Journal of neurooncology 118, 377-383, doi:10.1007/s11060-014-1443-0 (2014).

31. Wakabayashi, T. et al. JCOG0911 INTEGRA study: a randomized screening phase II trial of interferon $\beta$ plus temozolomide in comparison with temozolomide alone for newly diagnosed glioblastoma. Journal of neuro-oncology 138, 627-636, doi:10.1007/s11060-018-2831-7 (2018).

32. Takano, K. et al. Diagnostic and Prognostic Value of 11C-Methionine PET for Nonenhancing Gliomas. AJNR. American journal of neuroradiology, 37, 44-50 https://doi.org/10.3174/ajnr.A4460 (2016).

33. Bauer, E. K. et al. Prediction of survival in patients with IDH-wildtype astrocytic gliomas using dynamic 0-(2-[(18)F]-fluoroethyl)-L-tyrosine PET. European journal of nuclear medicine and molecular imaging, 47, 1486-1495 https://doi.org/10.1007/s00259-020-04695-0 (2020).

34. Patel, C. B. et al. (18)F-FDOPA PET and MRI characteristics correlate with degree of malignancy and predict survival in treatment-naïve gliomas: a cross-sectional study. Journal of neuro-oncology 139, 399-409, doi:10.1007/s11060-018-2877-6 (2018).

35. Arita, H. et al. A combination of TERT promoter mutation and MGMT methylation status predicts clinically relevant subgroups of newly diagnosed glioblastomas. Acta neuropathologica communications, 4, 79 https://doi.org/10.1186/s40478-016-0351-2 (2016).

36. Oughourlian, T. C. et al. Rate of change in maximum (18)F-FDOPA PET uptake and non-enhancing tumor volume predict malignant transformation and overall survival in low-grade gliomas. Journal of neuro-oncology 147, 135-145, doi:10.1007/s11060-020-03407-w (2020).

\section{Figures}




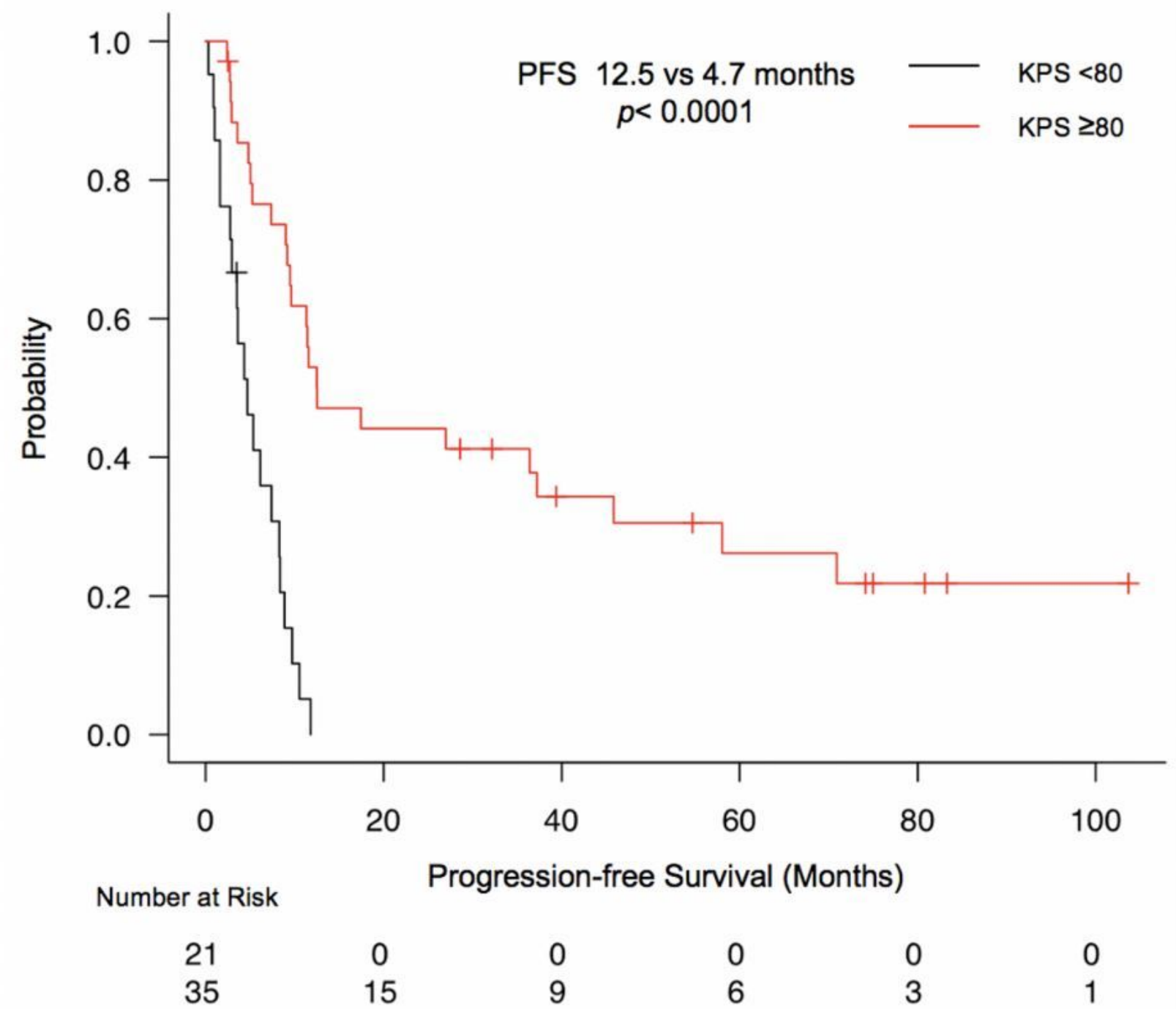

Figure 1

Kaplan-Meier plot of PFS in relation to preoperative KPS. 

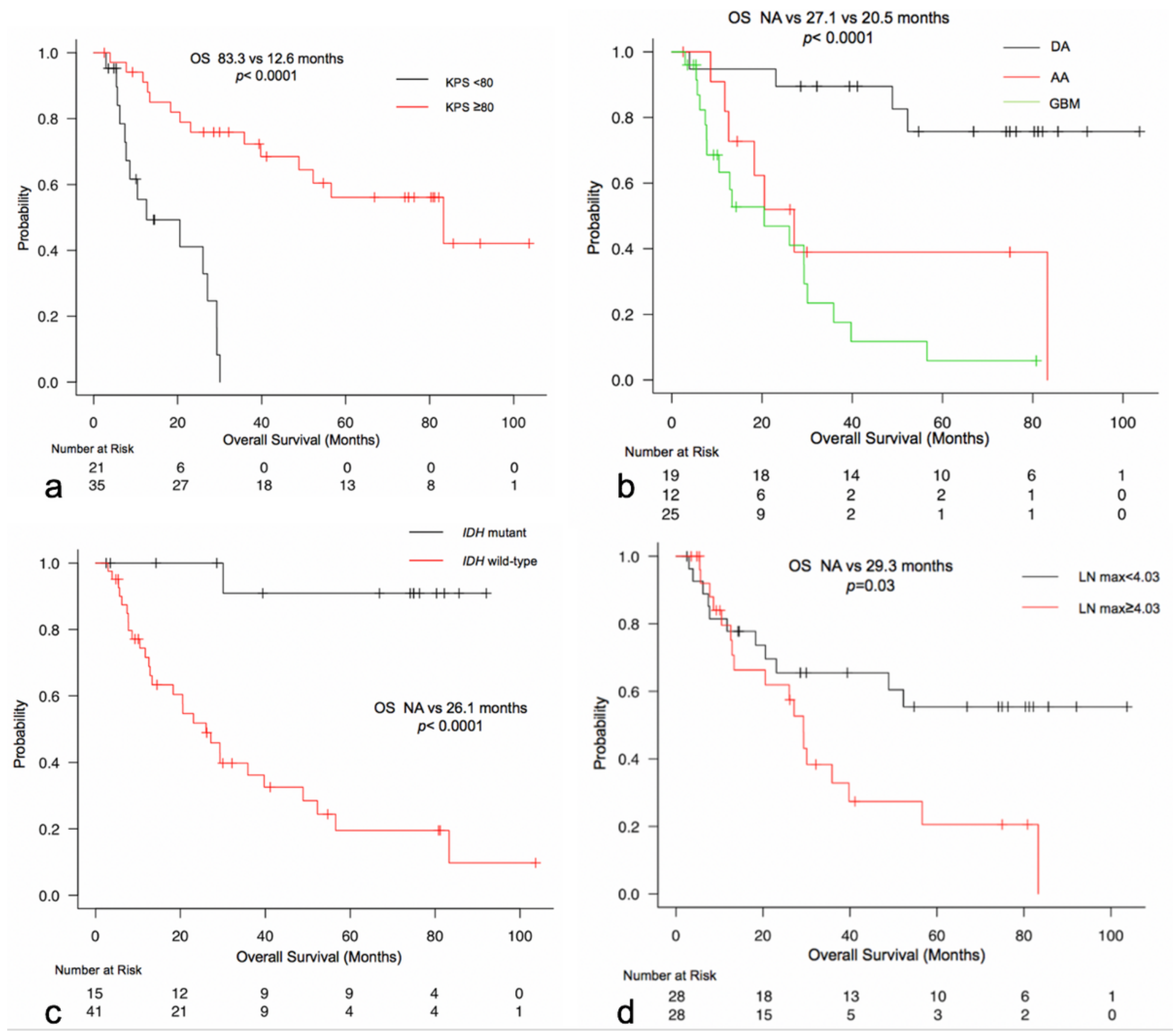

Figure 2

Kaplan-Meier plot of OS in relation to preoperative KPS (A), histology (B), IDH status (C), and LN max (D). 


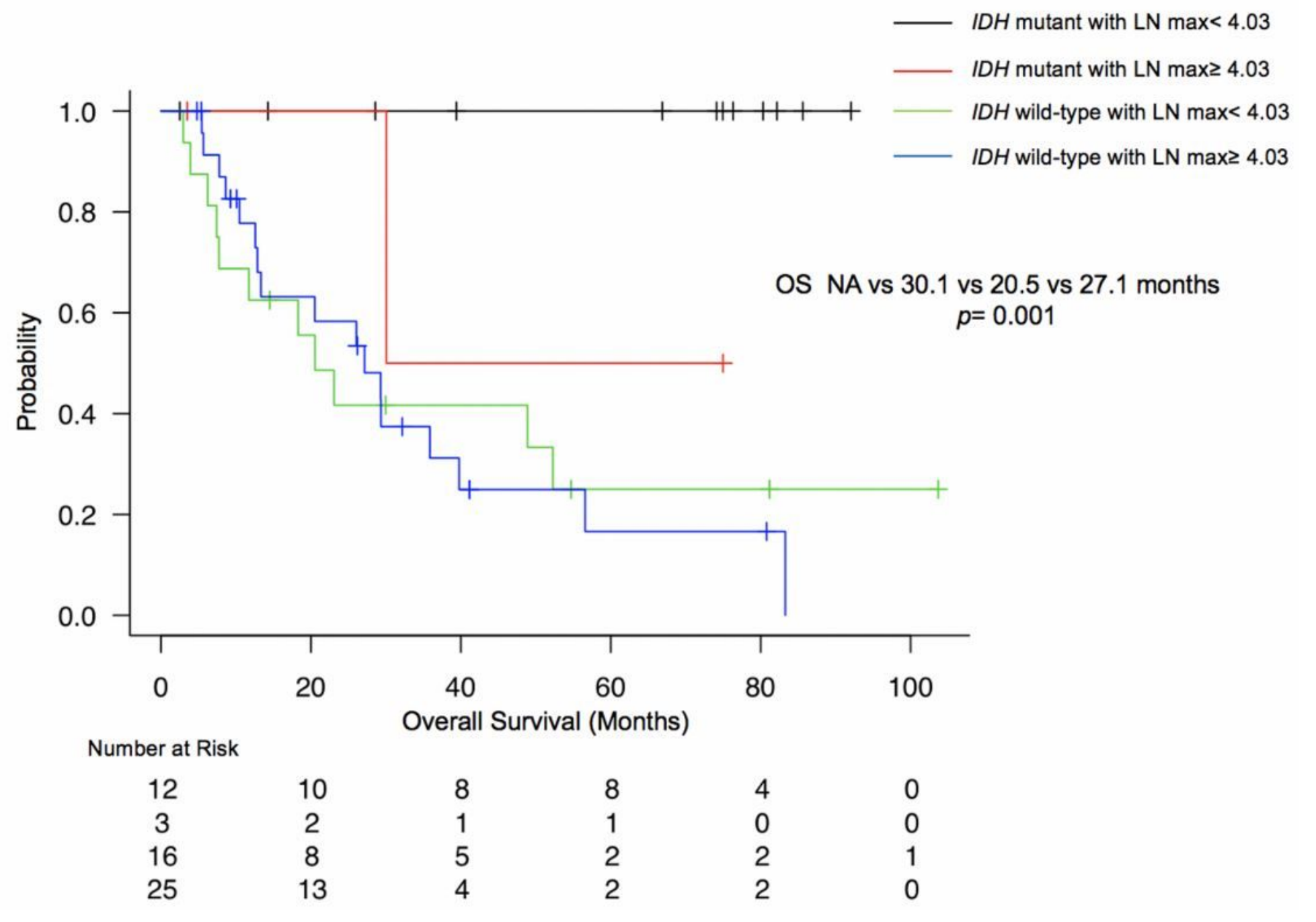

Figure 3

Kaplan-Meier plot of the OS in relation to the IDH status/LN max classification. A significant difference in OS existed between patients with IDH mutant/LN max $<4.03$ and those with IDH mutant/LN max $\geq 4.03$ $(p=0.034)$, although no significant difference in OS was evident between patients with IDH mutant/LN $\max \geq 4.03$ and those with IDH wild-type/LN max $<4.03(p=0.40)$, or between patients with IDH wildtype/LN max $<4.03$ and those with IDH wild-type/LN $\max \geq 4.03(p=0.84)$.

\section{Supplementary Files}

This is a list of supplementary files associated with this preprint. Click to download.

- floatimage1.png

- floatimage2.png

- floatimage3.png 